\title{
Effets pharmacologiques d'un extrait aqueux de Bidens pilosa L. (Asteraceae) sur le système cardiovasculaire de mammifères
}

\section{Léandre K. KOUAKOU', Flavien TRAORE ${ }^{2 *}$, Jean-Claude K. ABO ${ }^{2}$ et Etienne Ehovan EHILE ${ }^{1}$}

'Laboratoire de physiologie animale, Pharmacologie et pharmacopée, UFR Sciences de la nature, 02 BP 80I ABIDJAN 02, Université d'Abobo-Adjamé Côte-d'Ivoire

${ }^{2}$ Laboratoire de physiologie animale, UFR Biosciences, 22 BP 582 Abidjan 22, Université de Cocody-Abidjan, Côte-d'Ivoire

(Reçu le 13 Novembre 2006, accepté le 26 Avril 2007)

* Correspondance, courriel :

\section{Résumé}

L'extrait aqueux brut de Bidens pilosa L. (Asteraceae), dans un intervalle de concentrations compris entre $1 \times 10-3$ et 1,4x10-2 g/ $/ \mathrm{kg}$ p.c, provoque chez le Lapin une hypotension transitoire dose dépendante. En revanche, l'amplitude et la fréquence respiratoires sont peu affectées. Sur le cœur isolé de Rat, ce concentré naturel induit des effets inotrope et chronotrope négatifs à partir de $10-10 \mathrm{mg} / \mathrm{mL}$. Ces effets atteignent leur maximum à $10-8 \mathrm{mg} / \mathrm{mL}$ puis diminuent à $10-6 \mathrm{mg} / \mathrm{mL}$. A $10-4 \mathrm{mg} / \mathrm{mL}$ l'enregistrement obtenu est pratiquement normal.

Ces résultats suggèrent que l'hypotension provoquée par Bidens pilosa L. pourrait résulter d'une part des effets relaxants observés par certains auteurs sur le muscle lisse vasculaire et d'autre part sur les effets cardiodépresseurs des principes actifs contenus dans cette plante.

A l'aide de l'atropine qui est une substance antagoniste des récepteurs muscariniques, trois types de principes actifs ont été mis en évidence.

Il s'agit :

1) Des principes actifs cholinomimétiques de type muscarinique inhibés par l'atropine.

2) Des principes actifs cardiodépresseurs insensibles à l'atropine.

3) Des principes actifs cardioactivateurs actifs aux fortes doses de Bidens pilosa $\mathrm{L}$. A 1,3x10-1 g/kg p.c, l'extrait brut de Bidens pilosa L. provoque une profonde hypotension suivie d'un arrêt respiratoire létal pour le Lapin. Les résultats obtenus justifient 
l'utilisation de Bidens pilosa L. en Médecine traditionnelle pour traiter l'hypertension artérielle.

Mots-clés : Bidens pilosa L, acetylcholine, atropine, substances cholinomimétiques, cardiodepressantes et cardiactivatrices.

\section{Abstract \\ Pharmacological effects of an aqueous extract of Bidens pilosa $L$. (Asteraceae) on the cardiovascular system of mammals}

The crude aqueous extract of Bidens pilosa L. (Asteraceae), for concentrations ranging from $1 \times 10-3$ to $1.4 \times 10-2 \mathrm{~g} / \mathrm{kg}$ of body weight (b. w.) induced a transient dose dependant hypotension in the rabbit whereas the respiratory wide range and frequency were less affected. $0 \mathrm{n}$ the isolated heart of rat, this natural substance caused negative inotropic and chronotropic effects from the concentration of $10-10 \mathrm{mg} / \mathrm{mL}$. These effects reached their maximum values at $10-8 \mathrm{mg} / \mathrm{mL}$ and then decreased at $10-6 \mathrm{mg} / \mathrm{mL}$. At $10-4 \mathrm{mg} / \mathrm{mL}$, no major changes were noticed in the recordings.

These results suggest that the hypotension induced by Bidens pilosa L. could result, on the one hand, from the relaxing effects observed by some authors on the vascular smooth muscle and on the other hand, from the cardiodepressant effects of the active principles contained in the plant.

Three kinds of active principles were revealed in the presence of atropine, a muscarinic cholinoceptors antagonist. These are :

1) Muscarinic cholinomimetic active principles inhibited by atropine.

2) Cardiodepressant principles insensible to atropine.

3) Cardioactive principles acting at high doses of Bidens pilosa.

At the dose of $1.3 \times 10-1 \mathrm{~g} / \mathrm{kg}$ of b.w., the crude extract of Bidens pilosa caused a deep hypotension followed by a respiratory arrest lethal for the rabbit.

The results obtained justify the use of Bidens pilosa in traditional medicine to treat hypertension.

Keywords : Bidens pilosa, acetylcholine, atropine cholinomimetic, cardiodepressant and cardioactive substances. 


\section{Introduction}

Bidens pilosa L. est une plante herbacée, érigée, les folioles sont régulièrement dentées et glabres [1]. Espèce pantropicale très répandue depuis l'Angola jusqu'au Cameroun, elle est également rencontrée en Côte d'lvoire où elle envahit les anciennes cultures $[1,2]$.

Bidens pilosa $L$. renferme des essences et des composés polyacétyléniques [2,3]. Elle contient des substances tels que les flavonoïdes, l'acide linolénique, le phenylheptetriyne et des glucosides [4-6].

Cette plante est utilisée en Médecine traditionnelle africaine pour le traitement des différents troubles cardiaques en particulier l'hypertension artérielle. Bidens pilosa L. est également employée pour soigner la dysenterie et la diarrhée [1,2]. Elle calmerait les douleurs musculaires et les maux d'oreilles et passe pour être un nématicide. Elle est recommandée comme anti-poison, contre les morsures de Serpents, dans le traitement des ictères et de la variole. Elle aurait une action cicatrisante et calmerait les enfants agités $[1,2]$.

Les études pharmacologiques qui ont été effectuées sur Bidens pilosa L. ont révélé que les composés contenus dans cette plante ont des activités anti-microbiennes, antiinflammatoires [5,6], anti-bactériennes [7], anti-malariale [8], anti-ulcère gastrique [3,9] et anti-hyperglycémiantes [10]. Outre ces propriétés, les extraits de ce végétal ont des effets vasodilatateurs, hypotenseurs [11,12] et hépatoprotecteurs [13].

Les objectifs de ces travaux sont d'étudier les effets d'un extrait aqueux de Bidens pilosa L. (Asteraceae) sur le système cardiovasculaire ef la respiration de Mammifères, de caractériser pharmacologiquement les principes actifs contenus dans cet extrait et, d'établir, si possible, un lien entre les effets de ce concentré naturel et les usages traditionnels de cette plante médicinale africaine.

\section{Matériel et méthodes}

\section{2-1. Préparation de l'extrait aqueux de Bidens pilosa $L$. (Bp)}

Cent grammes $(100 \mathrm{~g})$ de poudre obtenue à partir de feuilles de Bidens pilosa, préalablement séchées à l'air libre et à la température ambiante, sont mixées pendant 24 heures dans deux litres d'eau distillée. La solution obtenue est filtrée sur du coton hydrophile et du papier Whatman. Deux litres d'eau distillée sont ajoutées au culot puis mixées pendant deux heures et filtrées également. 
Les filtrats sont évaporés sous vide à $60^{\circ} \mathrm{C}$ grâce à un évaporateur rotatif BUCHI. La pâte obtenue est séchée à l'étuve à $80^{\circ} \mathrm{C}$. Le produit obtenu est utilisé comme l'extrait brut de Bidens pilosa.

\section{2-2. Animaux}

\section{2-2-1. Les Lapins}

Les Lapins utilisés appartiennent à l'espèce Oryctolagus cuniculus (Leporidés). Ils proviennent de fermes d'élevage situées à Abobo dans une banlieve de la ville d'Abidjan. Aussi sont-ils acclimatés pendant une semaine à l'animalerie de l'Unité de Formation et de Recherche (UFR) des Sciences de la Nature de l'Université d'AboboAdjamé (Abidjan, Côte d'Ivoire) dans le but de les maintenir dans un état physiologique semblable avant l'expérimentation. Ils pèsent en moyenne $2,2 \pm 0,2 \mathrm{~kg}$.

\section{2-2-2. Les Rats}

Les Rats utilisés sont des Rats blancs de l'espèce Ratus norvegicus pesant entre 170 et $300 \mathrm{~g}$. Ils proviennent de l'animalerie du Laboratoire de Physiologie Animale de I'UFR Biosciences de l'Université de Cocody (Abidjan, Côte d'Ivoire).

\section{2-3. Techniques d'enregistrement}

Dispositif expérimental et technique d'enregistrement de la pression artérielle couplée à la respiration

Le dispositif expérimental utilisé pour l'enregistrement de la pression artérielle est un manomètre de LUDWIG qui comprend un tube en $U$ contenant du mercure. Sur la colonne de mercure de l'une des branches du manomètre repose un stylet inscripteur. L'autre branche du tube en U étant reliée à la carotide permet la transmission des variations de la pression sanguine du Lapin au stylet inscripteur.

L'enregistrement des mouvements respiratoires se fait grâce à l'appareil de BENEDICT dont le stylet inscripteur est relié à une capsule de Marey. Branchée en dérivation entre la sortie de l'appareil et la canule reliée à la trachée artère du Lapin, cette capsule manométrique transmet les variations de pression dues aux mouvements respiratoires de l'animal au stylet inscripteur.

Le Lapin est anesthésié par injection intra péritonéale d'ethyl-uréthane $40 \%$ à raison de $\mathrm{lg} / \mathrm{kg}$ de poids corporel. Sa carotide est mise à nue et intubée à l'aide du cathéter du 
dispositif de LUDWIG. Les variations de la pression sont transcrites à l'aide d'un stylet inscripteur sur le papier d'enregistrement recouvert de noir de fumée.

Quant à la trachée elle est également mise à nue et reliée à la canule de l'appareil de BENEDICT. Cette capsule manométrique reçoit les mouvements respiratoires et les inscrits simultanément à ceux de la variation de la pression artérielle sur le cylindre recouvert d'un papier induit de noir de fumée.

L'extrait de Bidens pilosa L. est dissout dans une solution de Mac Ewen puis injecté au Lapin par la veine saphène.

\section{2-4. Intubation de la veine saphène}

Le Lapin anesthésié est mis en décubitus dorsal. Sa partie postérieure est tournée sur le côté et la peau est incisée le long de la face externe de la cuisse. II apparaît un vaisseau très saillant qui sera mis à nu par une incision, c'est la veine saphène. Une hémisection est réalisée sur cette veine pour y introduire un cathéter qui permet d'injecter du Mac Ewen hépariné au Lapin. Le cathéter est fixé à l'aide d'une ligature et les substances pharmacologiques testées sont injectées au Lapin par cette veine, grâce à une seringue.

Technique d'enregistrement de l'activité mécanique du cœur isolé de Rat

Le dispositif expérimental utilisé pour l'enregistrement de l'activité mécanique du cœur isolé de Rat en perfusion est le même que celui décrit par EHILE [14].

Le Rat est anesthésié par injection intrapéritonéale d'ethyl-uréthane $20 \%$ à raison de $\mathrm{lg} / \mathrm{kg}$ de poids corporel. Il est ensuite placé sous respiration artificielle. On pratique une thoracotomie médiane et le péricarde est dégagé.

La crosse aortique est intubée avec une canule puis ligaturée de même que les artères collatérales. L'autre extrémité de la canule d'intubation est fixée à la sortie du robinet à voies multiple du dispositif de perfusion et le cœur isolé est perfusé avec la solution physiologique de type Mac Ewen ou avec la solution test.

L'apex du cœur est relié à un stylet inscripteur par une pince serre fine et un fil de tresse. Les contractions du cœur sont transmises au levier inscripteur dont l'extrémité frotte sur un cylindre recouvert de papier enduit de noir de fumée et entrâné en mouvement circulaire par un moteur.

\section{2-5. Traitement des résultats expérimentaux}

Les enregistrements effectués sur papier enfumé sont vernis puis scannés avant d'être inversés grâce aux logiciels Photo editor et Paint de Microsoft. L'analyse statistique et les courbes ont été réalisées grâce aux logiciels GraphPad Instat et GraphPad Prism (San 
Diego California, USA) respectivement. Le test ( $t$ ) de Student a été utilisé et les différences significatives sont marquées par une étoile (*) pour $p<0,05$.

\section{2-6. Solutions physiologiques}

Au cours de nos expériences, trois solutions ont été utilisées, la solution physiologique de référence, la solution de Mac Ewen et deux solutions modifiées les solutions hypercalcique (150\%) et hypocalcique $(50 \%)$.

La solution de Mac Ewen a la composition suivante (en mM) : $\mathrm{NaCl}, 130 ; \mathrm{KCl}, 5,6$; $\mathrm{CaCl} 2$, 2,16; NaH2P04, 0,91; $\mathrm{MgCl} 2,0,24$ et glucose, 11.

La solution hypercalcique 150 \% est une solution de Mac Ewen dont la concentration en $\mathrm{CaCl} 2$ est de 3,24 mM.

La solution hypocalcique $50 \%$ est une solution de Mac Ewen avec une concentration de $\mathrm{CaCl} 2$ de $1,08 \mathrm{mM}$.

NB: Les concentrations ioniques modifiées sont faibles de sorte que les changements d'osmolarité sont considérés comme négligeables.

\section{Résultats}

Effets dose-réponse de Bidens pilosa $\mathrm{L}$. (Bp) sur la pression artérielle et l'activité respiratoire de Lapin

La Figure $I$ présente un enregistrement type des effets de l'extrait aqueux brut de Bp à des doses croissantes allant de $1 \times 10-3$ à $1,4 \times 10-2 \mathrm{~g} / \mathrm{kg}$ p.c. sur la pression artérielle sanguine et la respiration de Lapin. L'extrait aqueux Bp est administré par voie intraveineuse.

La pression artérielle sanguine normale du Lapin est de $120 \mathrm{~mm} \mathrm{Hg}$ avec une respiration normale d'une amplitude de $20 \mathrm{~mm}$ et d'une fréquence de 76 cycles $/ \mathrm{mn}$.

L'injection d'une solution de Bp à des doses croissantes comprises entre lx10-3 et $1,4 \times 10-2 \mathrm{~g} / \mathrm{kg}$ p.c., induit des modifications de la pression artérielle, en fonction des doses injectées.

Pour des doses croissantes comprises entre $1 \times 10-3 \mathrm{~g} / \mathrm{kg}$ p.c ef $1,4 \times 10-2 \mathrm{~g} / \mathrm{kg} \mathrm{p.c,} \mathrm{on}$ enregistre des chutes de pression artérielle allant de 5,71 à $40 \mathrm{~mm} \mathrm{Hg}$, ce qui représente des diminutions respectives de 4,75 et $33,33 \%$ de la pression artérielle de référence. Après chaque chute la pression redevient normale à nouveau. 
A

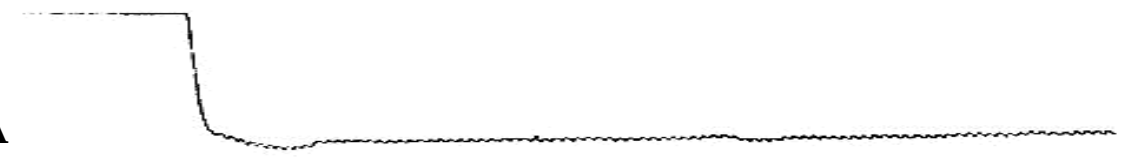

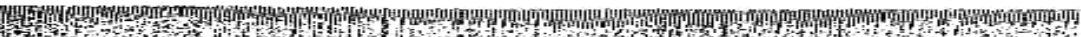

\section{$\uparrow \mathrm{Bp} 1 \times 10^{-3} \mathrm{~g} / \mathrm{kg}$}

B

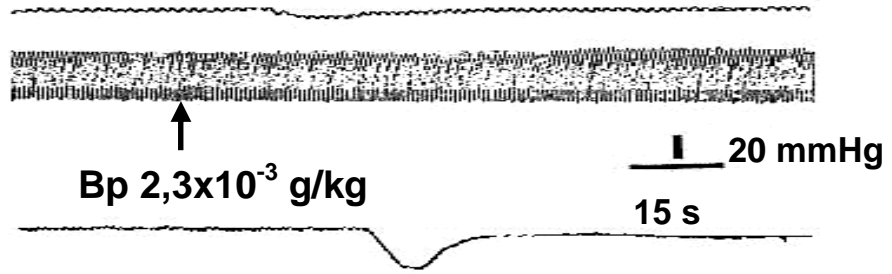

C

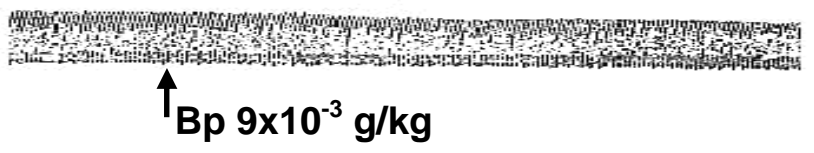

D

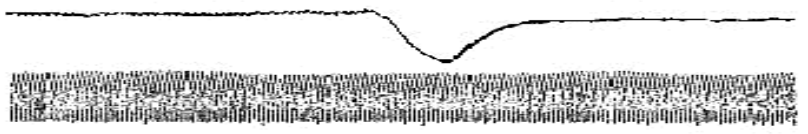

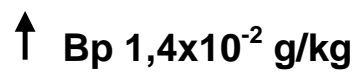

Figure 1 : Effet dose-réponse de Bidens pilosa (Bp) sur la pression artérielle et l'activité respiratoire chez le Lapin

Effets de Bp à : $1 \times 10-3 \mathrm{~g} / \mathrm{kg}$ p.c. ( $\mathrm{A}) ; 2,3 \times 10-3 \mathrm{~g} / \mathrm{kg}$ p.c. (B); $9 \times 10-3 \mathrm{~g} / \mathrm{kg}$ p.c. (C); $1,4 \times 10-2$ $\mathrm{g} / \mathrm{kg}$ p.c. (D).

La Figure 2 est un enregistrement type des effets de l'extrait aqueux brut de $B p$ à des fortes doses comprises entre $4,5 \times 10^{-2} \mathrm{~g} / \mathrm{kg}$ p.c et $1,3 \times 10^{-1} \mathrm{~g} / \mathrm{kg} \mathrm{p.c}$.

La pression artérielle normale du Lapin utilisé dans cette expérience est de $126 \mathrm{~mm}$ de $\mathrm{Hg}$ et la respiration normale a une amplitude de $20 \mathrm{~mm}$ et une fréquence égale à 88 cycles/mn.

A $4,5 \times 10^{-2} \mathrm{~g} / \mathrm{kg}$ p.c de $\mathrm{Bp}$, la pression artérielle chute de $78 \mathrm{~mm}$ de $\mathrm{Hg}$, valeur représentant une diminution de $61,90 \%$ par rapport à la pression normale. Cette hypotension est partiellement réversible. Les paramètres de la respiration restent inchangés.

A $1,3 \times 10^{-1} \mathrm{~g} / \mathrm{kg}$ p.c de Bp, la chute de la pression artérielle mesure $64 \mathrm{~mm}$ de $\mathrm{Hg}$, représentant une diminution de 50,79 \%. Cette hypotension irréversible entraine la mort de l'animal par arrêt respiratoire. 
A
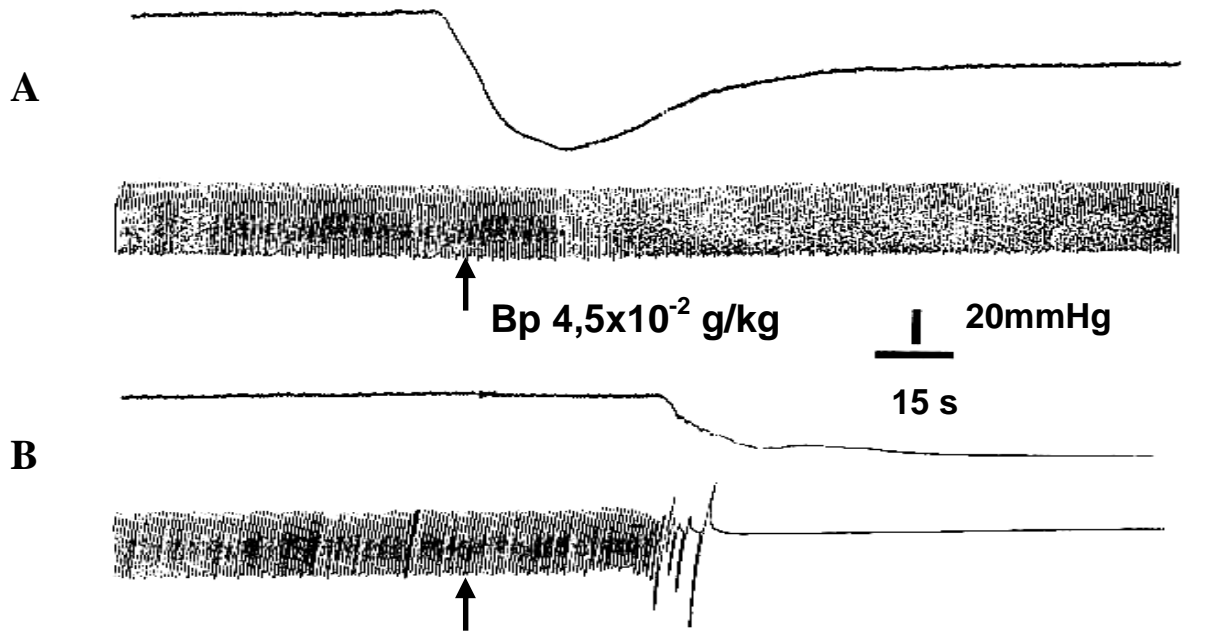

Bp $1,3 \times 10^{-1} \mathrm{~g} / \mathrm{kg}$

Figure 2 : Effets de fortes doses de Bp sur la pression artérielle et l'activité respiratoire chez le Lapin

Effets de Bp à: 4,5x10-2 g/kg p.c. (A); 1,3×10-1 g/kg p.c. (B)

Effets de Bidens pilosa L. sur la pression artérielle et l'activité respiratoire de Lapin en présence d'atropine.

La Figure 3 présente un enregistrement type des effets de l'injection intraveineuse d'un extrait aqueux brut de Bidens pilosa (Bp), à des doses croissantes allant de $1 \times 10^{-3}$ à $1,4 \times 10^{-2} \mathrm{~g} / \mathrm{kg}$ p.c sur la pression artérielle sanguine et la respiration de Lapin après une injection préalable d'atropine à $9 \times 10^{-6} \mathrm{~g} / \mathrm{kg}$ p.c.

La valeur de la pression artérielle normale du Lapin utilisé dans cette expérience est de $116 \mathrm{~mm} \mathrm{Hg}$ tandis que l'amplitude et la fréquence respiratoires du Lapin sont, respectivement, de $20 \mathrm{~mm}$ et de 60 cycles/minute.

L'injection d'atropine à $9 \times 10^{-6} \mathrm{~g} / \mathrm{kg}$ p.c. n'a aucun effet décelable sur la pression artérielle et sur les paramètres de la respiration.

Aux doses comprises entre $1 \times 10^{-3} \mathrm{~g} / \mathrm{kg}$ p.c et $2,3 \times 10^{-3} \mathrm{~g} / \mathrm{kg} \mathrm{p.c,} \mathrm{Bp} \mathrm{n'a} \mathrm{pas} \mathrm{d'effet} \mathrm{sur} \mathrm{la}$ pression artérielle et la respiration de Lapin en présence d'atropine. Entre $9 \times 10^{-3}$ ef $1,4 \times 10^{-2} \mathrm{~g} / \mathrm{kg} \mathrm{p}$.c Bp induit des hypotensions qui mesurent entre 20 et $22,85 \mathrm{~mm} \mathrm{Hg}$, ce qui représente des diminutions de la pression artérielle normale comprises entre 17,24 et $19,69 \%$. L'amplitude et la fréquence respiratoire restent pratiquement invariables au cours de cette expérience. 
A

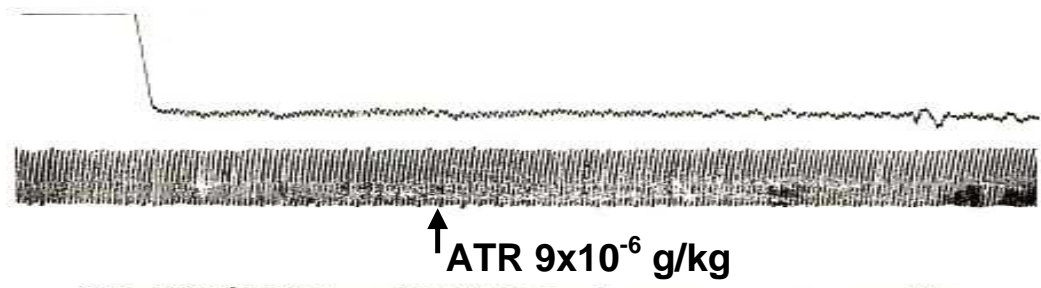

B

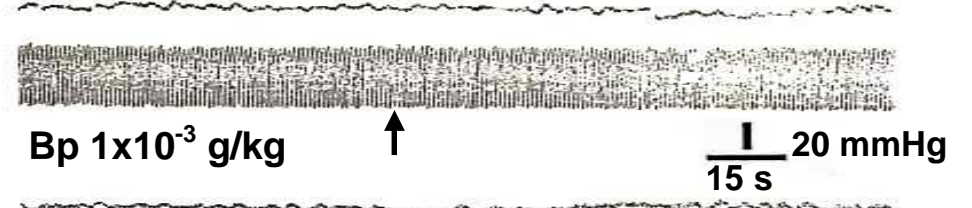

$\mathrm{C}$

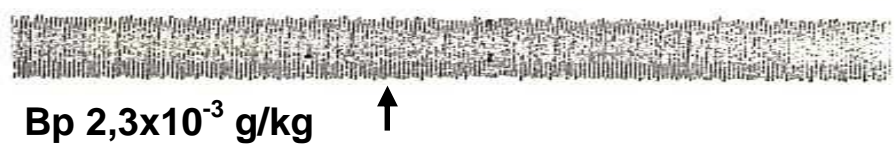

$\mathrm{D}$

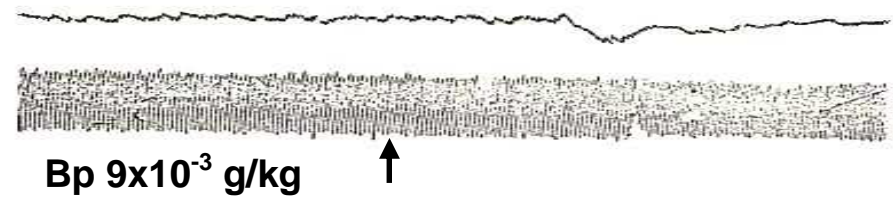

E

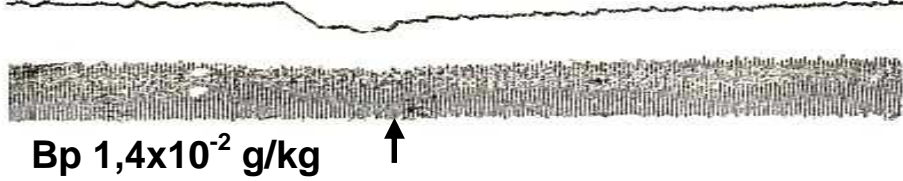

Figure 3 : Interaction Bp-Atropine (ATR) sur la pression artérielle et l'activité respiratoire chez le Lapin

Effets de l'atropine (ATR) à $9 \times 10-6 \mathrm{~g} / \mathrm{kg}$ p.c. (A) suivi des effets de $B p$ à: $1 \times 10-3 \mathrm{~g} / \mathrm{kg}$ p.c. (B); $2,3 \times 10-3 \mathrm{~g} / \mathrm{kg} \mathrm{p.c.} \mathrm{(C);} 9 \times 10-3 \mathrm{~g} / \mathrm{kg}$ p.c. (D); $1,4 \times 10-2 \mathrm{~g} / \mathrm{kg}$ p.c. (E)

Les résultats issus de 4 expériences identiques ont permis de tracer la courbe traduisant la variation de la pression artérielle en fonction de la dose de Bp en absence et en présence d'atropine (Figure 4).

De $1 \times 10^{-3}$ à $2,3 \times 10^{-3} \mathrm{~g} / \mathrm{kg}$ p.c, l'hypotension enregistrée est totalement inhibée $(100 \%)$ en présence d'atropine $9 \times 10^{-6} \mathrm{~g} / \mathrm{kg}$. De $2,3 \times 10^{-3} \mathrm{~g} / \mathrm{kg}$ p.c à $1,4 \times 10^{-2} \mathrm{~g} / \mathrm{kg}$ p.c, cette inhibition est partielle et varie entre $100 \%$ et $47,48 \%$. 


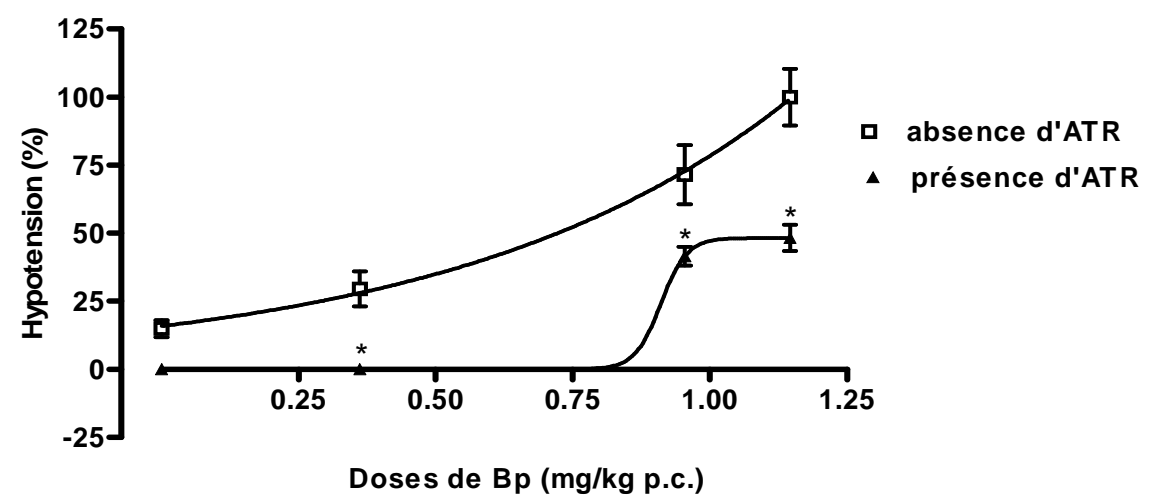

Figure 4 : Variation de la pression artérielle en absence et en présence d'atropine (ATR) en fonction des doses de $B p$

La Figure 5 est un enregistrement type des effets de $B p$ aux fortes doses comprises entre $4,5 \times 10^{-2} \mathrm{~g} / \mathrm{kg}$ p.c. et $1,3 \times 10^{-1} \mathrm{~g} / \mathrm{kg}$ p.c en présence d'atropine à $9 \times 10^{-6} \mathrm{~g} / \mathrm{kg} \mathrm{p}$.c. La valeur de la pression artérielle normale du Lapin utilisé dans cette expérience type est de $132 \mathrm{~mm}$ de $\mathrm{Hg}$ tandis que l'amplitude et la fréquence respiratoires du Lapin sont respectivement de $20 \mathrm{~mm}$ et de 67 cycles/minute.

En présence d'atropine (ATR) à $9 \times 10^{-6} \mathrm{~g} / \mathrm{kg}$, l'hypotension induite par $4,5 \times 10^{-2} \mathrm{~g} / \mathrm{kg} \mathrm{p} . c \mathrm{de}$ Bp est relativement faible $(28,57 \mathrm{~mm} \mathrm{Hg})$ et revient complètement à sa valeur de référence. Les paramètres de la respiration restent inchangés.

A
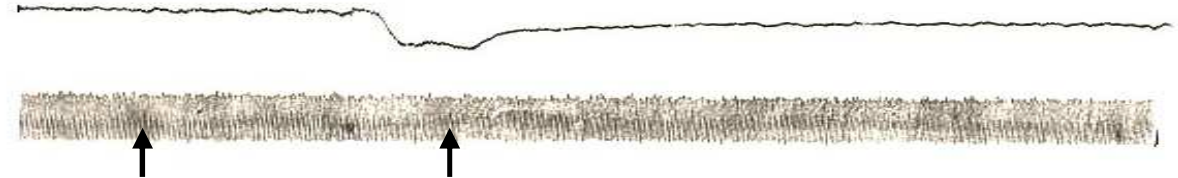

ATR $9 \times 10^{-6} \mathrm{~g} / \mathrm{kg} \quad$ Bp $4,5 \times 10^{-2} \mathrm{~g} / \mathrm{kg}$

I $20 \mathrm{mmHg}$

$15 s$

B

$$
\text { Tp } 1,3 \times 10^{-1} \mathrm{~g} / \mathbf{k g}
$$

Figure 5 : Effets de fortes doses de Bp sur la pression artérielle et l'activité respiratoire chez le lapin en présence d'atropine (ATR).

Effets de l'atropine à $9 \times 10-6 \mathrm{~g} / \mathrm{kg}$ p.c. (lère flèche) suivis des effets de $B p$ à 4,5x10-2 $\mathrm{g} / \mathrm{kg}$ p.c. (2ème fhèche) (A) et des effets de Bp à $1,3 \times 10-1 \mathrm{~g} / \mathrm{kg}$ p.c. (B) 
L'hypotension observée à $1,3 \times 10^{-1} \mathrm{~g} / \mathrm{kg}$ p.c de Bp ne mesure plus que $42,85 \mathrm{~mm} \mathrm{Hg}$ en présence d'atropine $9 \times 10^{-6} \mathrm{~g} / \mathrm{kg}$. Elle retourne à la normale. Toutefois, cette hypotension s'accompagne d'une nette diminution de la fréquence respiratoire.

\section{Effets de Bp sur l'activité mécanique du cœur isolé de rat}

La Figure 6 est un enregistrement type de l'activité contractile du cœur isolé de Rat perfusé avec des concentrations croissantes de Bp.

Pour une faible concentration égale à $10^{-10} \mathrm{mg} / \mathrm{mL}$ nous avons une diminution respective de l'amplitude de $45 \%$ et de la fréquence des contractions cardiaques de $30 \%$. Ces effets sont maximum à $10^{-8} \mathrm{mg} / \mathrm{mL}$. Ils diminuent de $83 \%$ pour l'amplitude et $67 \%$ pour la fréquence de contraction lorsque le cœur est perfusé à une concentration de $10^{-6}$ $\mathrm{mg} / \mathrm{mL}$. Par contre pour une plus forte concentration de $10^{-4} \mathrm{mg} / \mathrm{mL}$, la fréquence et l'amplitude cardiaque diminuent respectivement de $28 \%$ et $26 \%$.

Nous observons un retour aux valeurs normales lorsque nous perfusons à nouveau de la solution physiologique de Mac Ewen. Ces effets réversibles sont observables 30 s après un retour en milieu normal.

A

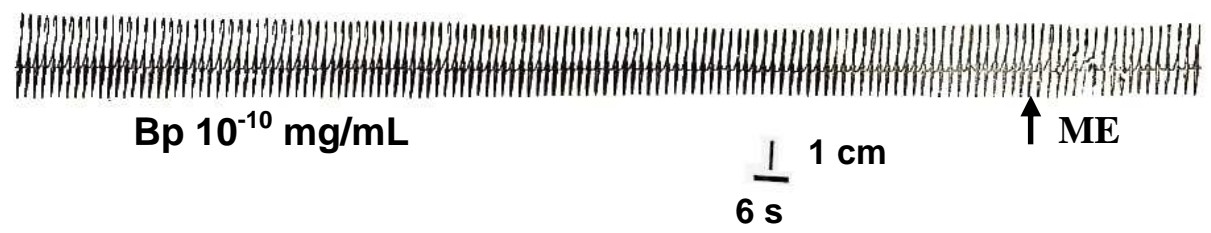

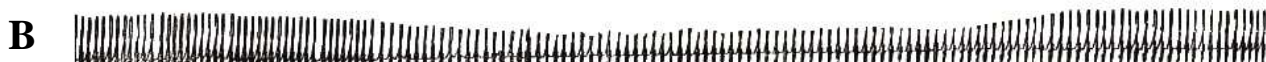

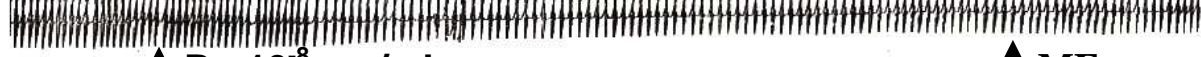
† Bp $10^{-8} \mathrm{mg} / \mathrm{mL} \quad$ † $\mathrm{ME}$

C

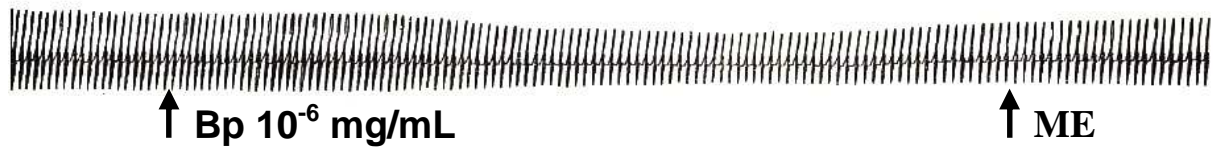

D

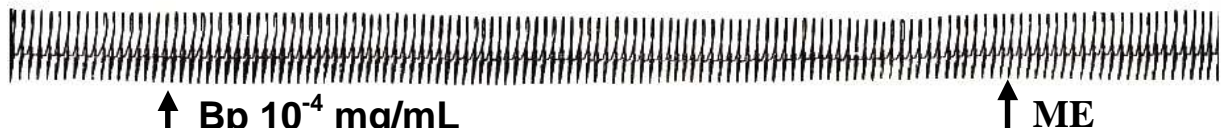
$\uparrow \mathrm{Bp} 10^{-4} \mathrm{mg} / \mathrm{mL}$

Figure 6 : Effet dose-réponse de Bp sur l'activité mécanique du caur isolé de Rat

Effets de Bp (lère flèche) à: $10-10 \mathrm{mg} / \mathrm{mL}(A) ; 10-8 \mathrm{mg} / \mathrm{mL}(B) ; 10-6 \mathrm{mg} / \mathrm{mL}(C) ; 10-4$ $\mathrm{mg} / \mathrm{mL}$ (D) suivis du retour dans le Mac Ewen (ME) (2ème fleche). 
Les résultats obtenues dans les mêmes conditions à partir d'une répétition d'expérimentations $(\mathrm{n}=3$ ) ont permis de construire les graphiques des Figures $7 \boldsymbol{A}$ et $\boldsymbol{T B}$ montrant l'évolution de l'amplitude et de la fréquence cardiaque pour les concentrations allant de $10^{-10}$ à $10^{-4} \mathrm{mg} / \mathrm{mL}$.
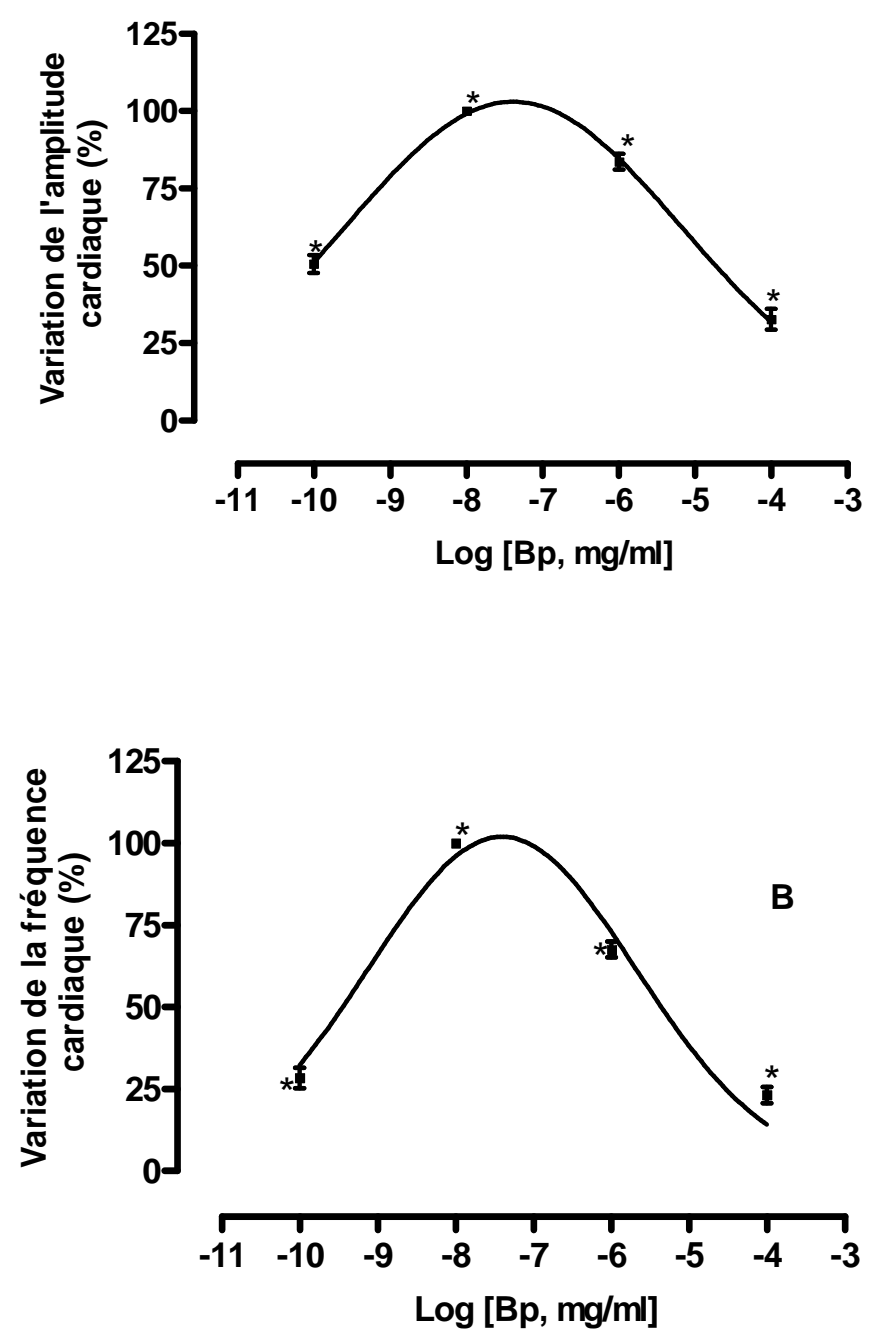

Figure 7 : Variation de l'amplitude (A) et de la fréquence cardiaque (B) en fonction des concentrations de $B p$

Effet de l'extrait aqueux brut de Bidens pilosa L. en présence d'atropine sur l'activité mécanique du cœur isolé de rat. 
Pour réaliser cette étude, nous avons testé Bidens pilosa à trois concentrations $: 10^{-10}$ $\mathrm{mg} / \mathrm{mL}, 10^{-8} \mathrm{mg} / \mathrm{mL}$ ef $10^{-6} \mathrm{mg} / \mathrm{mL}$ en absence et en présence d'atropine à $10^{-4} \mathrm{mg} / \mathrm{mL}$ (Figure 8).

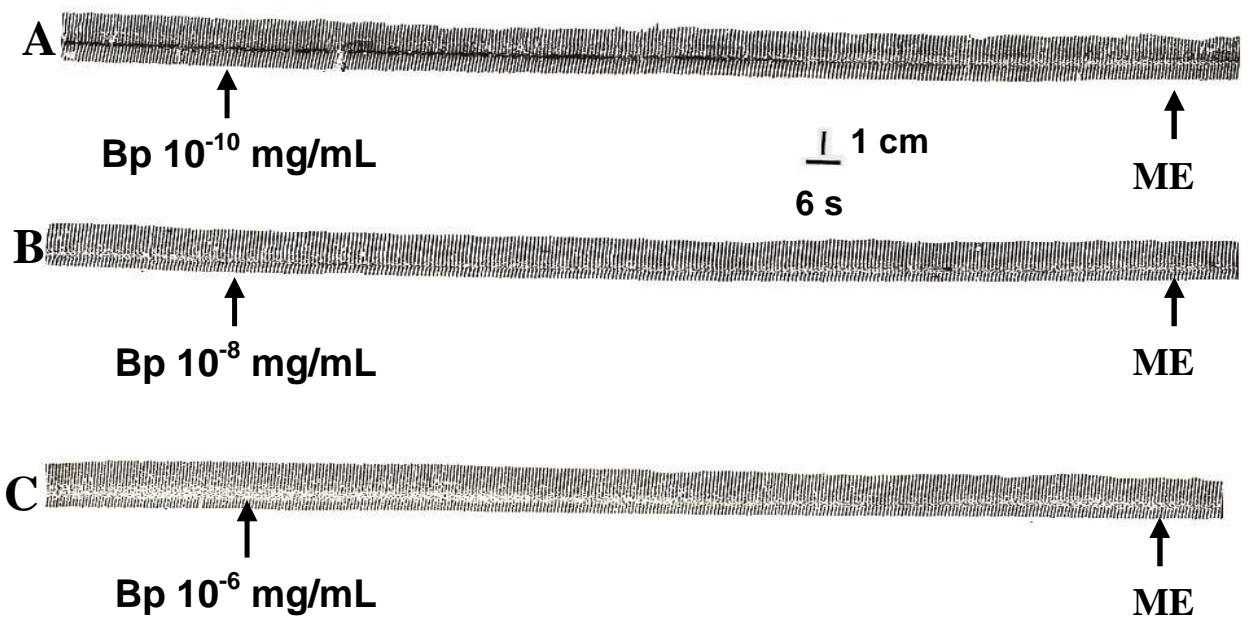

Figure 8 : Effets de Bp sur l'activité mécanique du caur isolé de Rat en absence d'ATR.

Effets de $B p$ à $10^{-10}(A), 10^{-8}(B)$ et $10^{-6}(C) \mathrm{mg} / \mathrm{mL}$ suivi du retour dans le Mac Ewen (ME) (2 $2^{\text {ème }}$ flèche).

Les effets de Bp dans les conditions normales pour les concentrations allant de $10^{-10}$ à $10^{-4} \mathrm{mg} / \mathrm{mL}$ ont été déjà décrits précédemment. Un pré-traitement du tissu cardiaque avec de l'atropine réduit l'effet de Bp. En effet, en présence d'atropine à $10^{-4} \mathrm{mg} / \mathrm{mL}$ (Figure 9), l'amplitude de la contraction reste inchangée pour les trois concentrations choisies. Quant à la fréquence, elle diminue faiblement. Ainsi, à $10^{-10}, 10^{-8}$ ef $10^{-6} \mathrm{mg} / \mathrm{mL}$, la fréquence diminue respectivement de $29 \%, 27 \%$ et $24 \%$.

Les Figures $10 A$ et $10 B$ sont des représentations graphiques $(n=3)$ qui traduisent les variations respectives de la fréquence cardiaque et de l'amplitude en fonction de concentrations croissantes de Bp en absence et après un pré-traitement du cœur isolé à l'atropine à une concentration de $10^{-4} \mathrm{mg} / \mathrm{mL}$. 
A

5.

ATR $10^{-4} \mathrm{mg} / \mathrm{mL} \quad$ Bp $10^{-10} \mathrm{mg} / \mathrm{mL}$

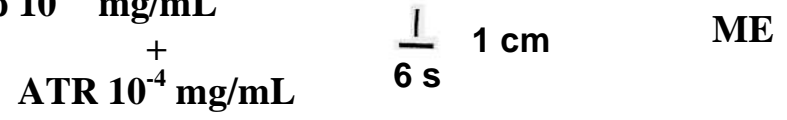

B

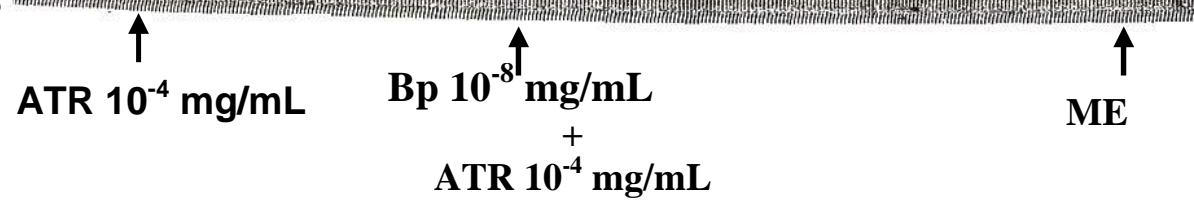

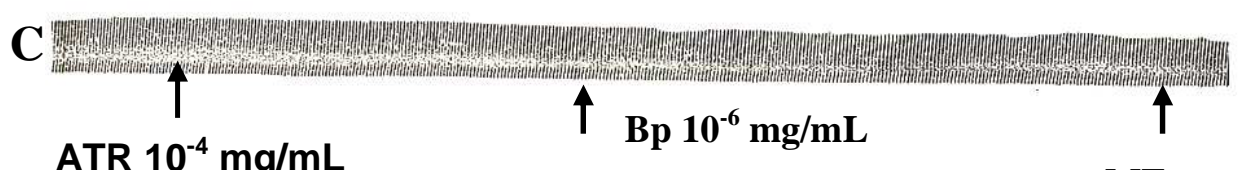

$\stackrel{+}{\operatorname{ATR} 10^{-4} \mathrm{mg} / \mathrm{mL}}$

ME

Figure 9 : Interaction Bp-atropine (ATR)sur l'activité mécanique du caur isolé de Rat.

A: Effets de l'ATR 10-4 mg/mL (lère flèche) suivis des effets de Bp à 10-10 mg/mL (2ème flèche) et du retour dans le Mac Ewen (ME) (3ème flèche)

B: Effets de l'ATR 10-4 mg/mL (lère flèche) suivis des effets de Bp à 10-8 mg/mL (2ème flèche) et du retour dans le Mac Ewen (ME) (3ème flèche)

C: Effets de l'ATR 10-4 mg/mL (lère flèche) suivis des effets de Bp à 10-6 mg/mL (2ème flèche) et du retour dans le Mac Ewen (ME) (3ème flèche)

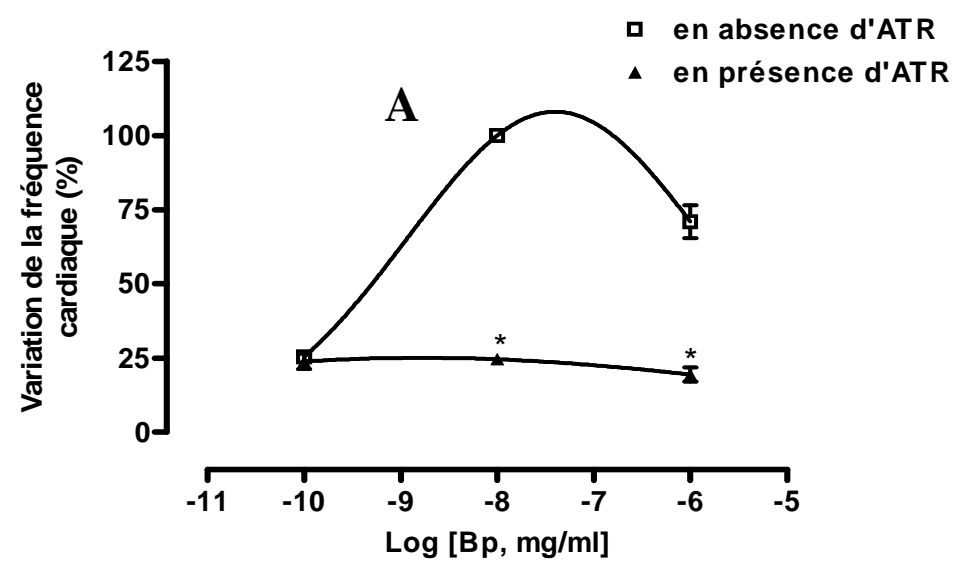

Léandre K. KOUAKOU et al. 


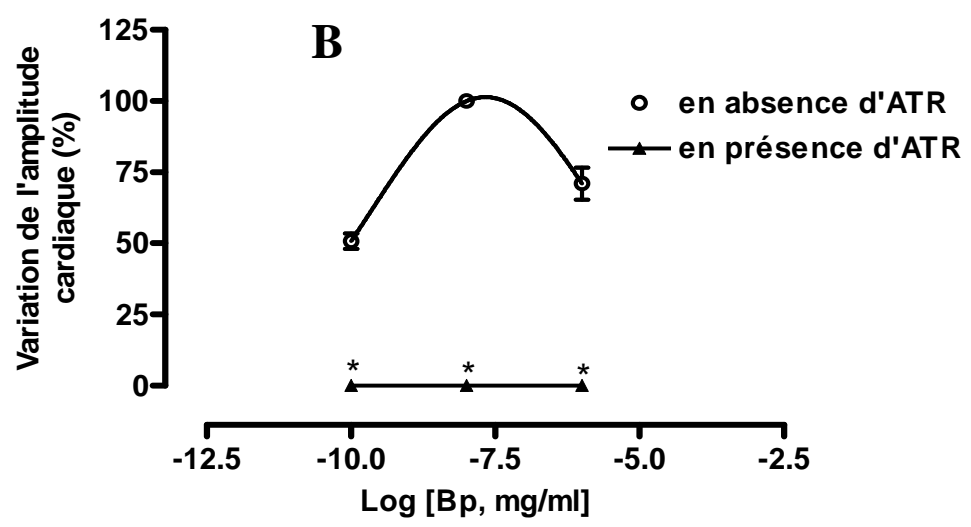

Figure 10 : Variation de la fréquence cardiaque (A) et de l'amplitude (B) en absence et en présence d'atropine (ATR), en fonction des concentrations de $B p$

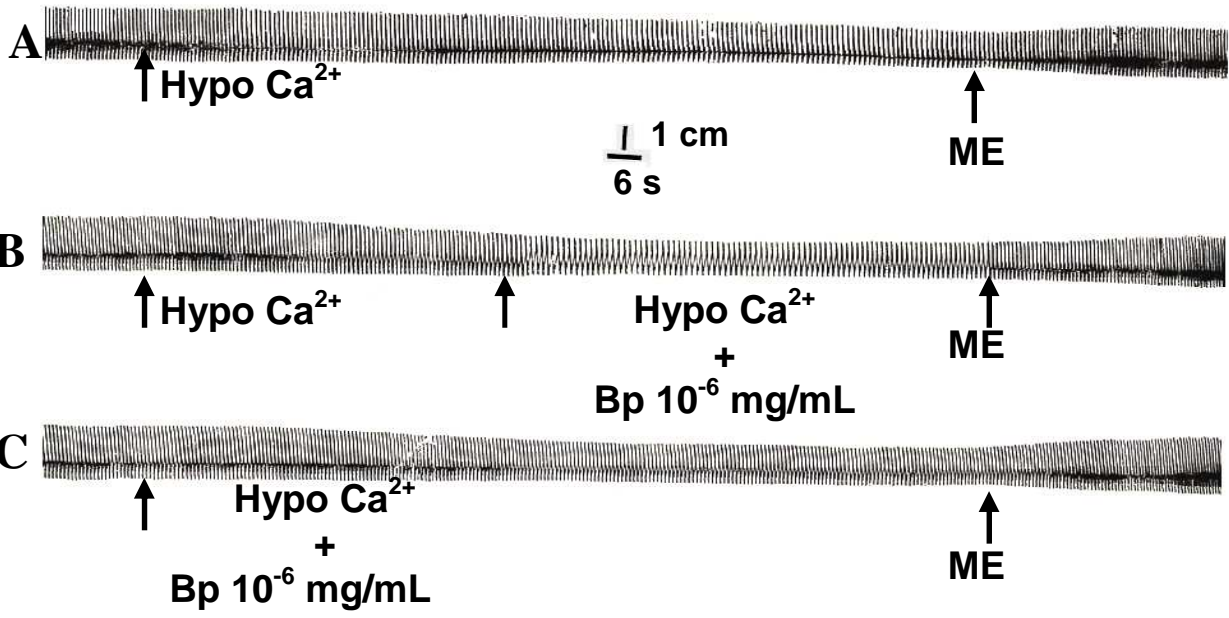

Figure 11 : Effets de Bp sur l'activité mécanique du caur isolé de Rat en milieu hypocalcique

A: Effets du milieu hypocalcique (lère flèche) suivi du retour dans le Mac Ewen (2ème flèche)

B: Effets du milieu hypocalcique (lère flèche) suivis des effets de Bp à $10-6 \mathrm{mg} / \mathrm{mL}$ (2ème flèche) et retour dans le Mac Ewen (ME) (3ème fèche)

C: Effets du milieu hypocalcique et de Bp à $10-6 \mathrm{mg} / \mathrm{mL}$ (lère flèche) suivi du retour dans le Mac Ewen (2ème flèche). 
A

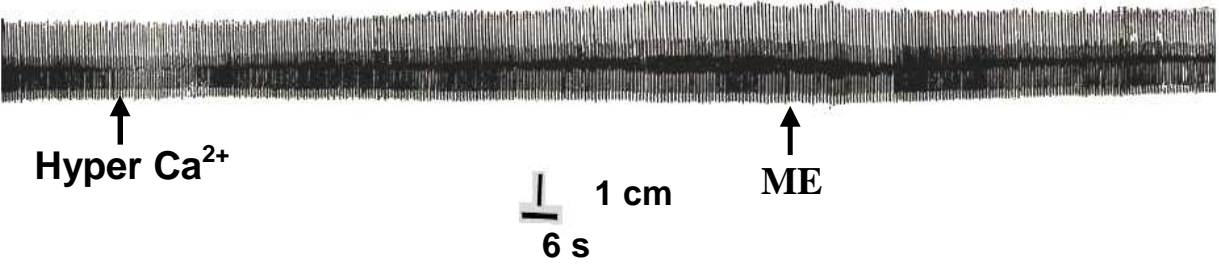

B

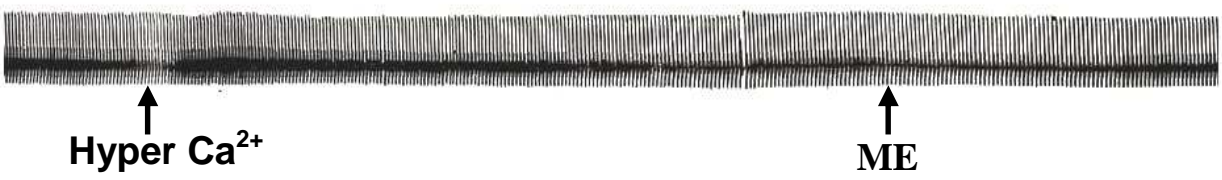

Bp $10^{-6} \mathrm{mg} / \mathrm{mL}$

Figure 12 : Effets de Bp sur l'activité mécanique du cœur isolé de Rat en milieu hypercalcique

A: Effets du milieu hypercalcique (lère flèche) suivi du retour dans le Mac Ewen (ME) (2ème flèche)

$B$ : Effets du milieu hypercalcique et de $B p$ à $10-6 \mathrm{mg} / \mathrm{mL}$ (lère flèche) suivi du retour dans le Mac Ewen (ME) (2ème flèche)

\section{Discussion}

L'extrait aqueux brut de Bidens pilosa L. (Asteraceae) induit une hypotension transitoire de type dose dépendante. Entre $1 \times 10^{-3}$ et $1,4 \times 10^{-2} \mathrm{~g} / \mathrm{kg} \mathrm{p.c,} \mathrm{on} \mathrm{observe} \mathrm{une} \mathrm{hypotension}$ totalement réversible. A $4,5 \times 10^{-2} \mathrm{~g} / \mathrm{kg}$ p.c, l'hypotension est profonde et se maintient partiellement. Jusqu'à cette valeur de concentration, l'amplitude et la fréquence respiratoires ne sont pas significativement affectées. La mort survient à la dose de $1,3 \times 10^{-1} \mathrm{~g} / \mathrm{kg}$ p.c à la suite d'une hypotension profonde de $64 \mathrm{~mm}$ de $\mathrm{Hg}$. Celle-ci est précédée d'une baisse de l'amplitude respiratoire suivie d'une apnée.

L'arrêt respiratoire semble donc étroitement lié à la surdose de Bp puisque l'extrait brut aqueux n'affecte nullement les paramètres de la respiration à des doses plus faibles.

L'hypotension dose dépendante induite par Bp rappelle les effets d'extraits aqueux de Musanga cecropioides (Cecropiaceae) [15], de Mareya micrantha (Euphorbiaceae) [16], de Lantana camara (Verbenaceae) [17] et d'extrait méthanolique de Bidens pilosa [12]. 
GUEDE Guina et al. [18] et $A B O$ et al. [16] ayant démontré l'existence de substances cholinomimétiques, dans $M$. micrantha,. il nous a semblé utile d'utiliser un antagoniste compétitif des récepteurs cholinergiques muscariniques tel que l'atropine pour caractériser, de façon préliminaire, les principes actifs contenus dans l'extrait brut de Bp.

En présence d'atropine à $9 \times 10^{-6} \mathrm{~g} / \mathrm{kg}$ p.c., les hypotensions observées entre $1 \times 10^{-3}$ et $2,3 \times 10^{-3} \mathrm{~g} / \mathrm{kg}$ p.c de Bp sont totalement inhibées. Celles enregistrées à $9 \times 10^{-3} \mathrm{~g} / \mathrm{kg} \mathrm{p.c,}$ $1,4 \times 10^{-2} \mathrm{~g} / \mathrm{kg} \mathrm{p}$.c et $4,5 \times 10^{-2} \mathrm{~g} / \mathrm{kg} \mathrm{p}$.c le sont partiellement, tandis que Bp à $1,3 \times 10^{-1} \mathrm{~g} / \mathrm{kg}$ p.c n'est plus mortelle. A cette dernière concentration, Bp provoque une profonde hypotension qui retourne au niveau normal. Cet effet s'accompagne simplement d'une baisse de la fréquence respiratoire.

L'action antagoniste de l'atropine a été également observée sur des hypotensions induites par des extraits aqueux bruts des feuilles de Musanga cecropioides [15] et de Mareya micrantha [16].

Nos résultats suggèrent, fortement, que l'extrait brut de Bidens pilosa comme ceux de Musanga cecropioides et de Mareya micrantha contiennent des substances cholinomimétiques de type muscarinique. En effet, il est connu que l'Ach induit, sur la pression artérielle, une hypotension dose dépendante [19]. Cette hypotension observée à $5 \times 10^{-6} \mathrm{~g} / \mathrm{kg} \mathrm{p}$.c chez le Lapin à l'aide du dispositif de Ludwig est totalement inhibée par l'ATR à $5 \times 10^{-4} \mathrm{~g} / \mathrm{kg}$ p.c selon $A B O$ et al. [16], ce qui indique que les effets de l'Ach sont médiés par les récepteurs muscariniques.

II est donc probable qu'il y ait une compétition entre les principes para sympathicomimétiques contenus dans l'extrait brut de Bidens pilosa et l'atropine sur

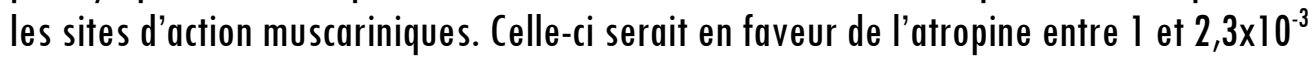
$\mathrm{g} / \mathrm{kg}$ p.c de Bp, ce qui explique qu'à ces doses, I'hypotension est complètement inhibée. A des doses de plus en plus élevées de $B p$, on assiste par contre à une inhibition de plus en plus faible de l'hypotension induite par l'extrait aqueux brut. Cet effet inverse indique que la compétition évolue en faveur de Bidens pilosa au fur et à mesure que sa concentration augmente.

L'hypotension provoquée par Bp pourrait être due aux effets relaxants dose-dépendant observés par DIMO [11] sur le muscle lisse aortique de Rat. II en résulterait en effet une diminution des résistances vasculaires. Mais cet effet hypotenseur de l'extrait aqueux brut de Bidens pilosa pourrait être aussi en relation avec les effets cardiodépresseurs des principes cholinomimétiques contenus dans ce concentré de source naturelle.

Pour cela, nous avons entrepris l'étude de l'effet de Bp sur l'activité mécanique du cœur isolé de Rat. 
L'extrait brut aqueux de Bidens pilosa induit des effets inotrope et chronotrope négatifs sur le cœur isolé de Rat à partir de $10^{-10} \mathrm{mg} / \mathrm{ml}$. Ces effets atteignent leur maximum à $10^{-}$ ${ }^{8} \mathrm{mg} / \mathrm{ml}$ puis diminuent à $10^{-6} \mathrm{mg} / \mathrm{ml}$. A $10^{-4} \mathrm{mg} / \mathrm{ml}$ l'enregistrement obtenu est pratiquement normal. Les effets chronotrope et inotrope négatifs observés entre $10^{-10}$ et $10^{-4} \mathrm{mg} / \mathrm{ml}$ de Bp sont parfaitement réversibles.

Ces résultats sur le cœur isolé de Rat renforcent l'hypothèse selon laquelle l'hypotension induite par l'extrait brut aqueux est liée, au moins en partie, à une cardiodépression. Ils sont parfaitement compatibles avec la présence de principes actifs cholinomimétiques dans l'extrait aqueux de Bidens pilosa.

Ces effets négatifs s'estompent à $10^{-6} \mathrm{mg} / \mathrm{mL}$ et disparaissent quasiment à $10^{-4} \mathrm{mg} / \mathrm{mL}$ du fait de l'existence probable, dans le même extrait brut, de substances ayant des effets contraires sur l'activité cardiaque de Rat. En effet, la présence de principes actifs induisant aux concentrations élevées un effet chronotrope et inotrope positif marqués pourraient supprimer les effets négatifs induits par $B p$, donnant ainsi à $10^{-4} \mathrm{mg} / \mathrm{mL}$, l'allure d'un enregistrement normal.

L'effet des seconds principes actifs serait plus marqué aux concentrations élevées, certainement à cause de leur faible concentration dans l'extrait aqueux brut de Bidens pilosa. Les effets chronotrope et inotrope négatifs induits par Bp rappellent ceux de l'acétylcholine sur le cœur [20]. Les effets de l'Ach étant entièrement médiés par les récepteurs muscarimiques [20] nous avons étudié l'interaction Bp-atropine sur le cœur isolé de Rat.

L'atropine inhibe entièrement les effets inotropes négatifs induits par $\mathrm{Bp}$ entre $10^{-10}$ $\mathrm{mg} / \mathrm{mL}$ et $10^{-6} \mathrm{mg} / \mathrm{mL}$. En revanche, les effets chronotropes négatifs ne sont que partiellement affectés même s'ils sont fortement réduits.

Ces résultats montrent que les effets des substances cholinomimétiques sur le cœur de Rat sont médiés par les récepteurs muscariniques.

Il faut donc admettre que l'hypotension induite par Bp est liée aux effets conjugués de la cardiodépression et de la vasodilatation induite par les principes cholinomimétiques.

Les récepteurs cholinergiques cardiaques étant de type muscarinique, on peut suggérer que les effets chronotropes négatifs, insensibles à l'ATR, sont liés à l'existence de principes actifs cardiodépresseurs différents des substances cholinomimétiques.

Ainsi, ces résultats permettent de suggérer l'existence d'au moins 3 types de substances dans l'extrait brut aqueux de Bidens pilosa.

$1^{\circ}$ ) Les principes actifs cholinomimétiques de type muscarinique inhibés par l'ATR.

$2^{\circ}$ ) Les principes actifs cardiodépresseurs insensibles à l'ATR.

$3^{\circ}$ ) Les principes actifs cardioactivateurs actifs aux fortes doses de Bp.

L'activité mécanique du cœur isolé de Rat, sous-tendue par l'activité électrique, est 
tributaire des mouvements ioniques à travers la membrane myocardique. Pour cela, il importe d'étudier l'effet de Bp dans des milieux physiologiques modifiés afin d'apprécier l'implication des ions en cause dans son mécanisme d'action.

L'extrait brut aqueux de Bidens pilosa accentue les effets inotrope et chronotrope négatifs induits par le milieu hypocalcique sur le cœur isolé de Rat. Par contre, il atténue la fréquence et même supprime totalement les effets inotropes positifs du milieu hypercalcique sur la même préparation.

Ces résultats indiquent que $B p$ a les mêmes effets que le milieu hypocalcique sur le cœur isolé de Rat. Ces effets sont liés à la présence de substances cardio-dépressives dans l'extrait aqueux de Bidens pilosa en particulier les substances cholionomimétiques antagonisées par l'atropine.

Il est connu que dans l'oreillette, l'Ach active le courant potassique et diminue le courant calcique [21-23]. Les effets chronotrope et inotrope négatifs induits par Bidens pilosa pourraient donc résulter soit d'une baisse du flux entrant de calcium, soit d'une augmentation du flux sortant de potassium [24].

La suppression de l'augmentation de l'amplitude des contractions cardiaques en milieu hypercalcique où le courant entrant calcique devrait augmenter, indique clairement que l'action de Bidens pilosa pourrait être médiée par ce flux ionique [25].

L'utilisation ultérieure d'inhibiteurs spécifiques de différentes conductances ioniques pourrait nous éclairer, davantage, sur le mécanisme d'action de cet extrait.

\section{Conclusion}

Nos résultats montrent que l'extrait aqueux brut de Bidens pilosa induit sur la pression artérielle de Lapin une hypotension dose-dépendante à des faibles doses comprises entre $1 \times 10-3$ et $1,4 \times 10-2 \mathrm{~g} / \mathrm{kg} \mathrm{p.c}$, sans affecter l'activité respiratoire. A forte dose, $1,3 \times 10-1 \mathrm{~g} / \mathrm{kg}$ p.c, l'extrait induit une hypotension soutenue conduisant à un arrêt cardiaque et à un arrêt respiratoire. Ces effets sont partiellement inhibés par l'atropine.

Sur le cœur isolé de Rat, ce concentré naturel provoque des effets inotrope et chronotrope négatifs avec un effet maximum correspondant à la concentration de 10-8 $\mathrm{mg} / \mathrm{mL}$.

L'utilisation de l'atropine, un inhibiteur compétitif des récepteurs muscariniques, nous a permis de suggérer 3 types de principes actifs:

-des principes actifs cholinomimétiques muscariniques inhibés par l'ATR.

-des principes actifs cardiodépressifs insensibles à l'ATR auxquels s'ajoutent

-des principes actifs non cardiodépressifs.

L'utilisation de Bidens pilosa en médecine traditionnelle pour traiter l'hypertension 
artérielle, trouve donc sa justification dans les résultats exposés ci-dessus qui corroborent ceux de DIMO et al. [11,12].

En tout état de cause, une utilisation rationnelle de ce produit nécessite des analyses plus poussées à partir d'extraits fractionnés pour isoler et caractériser les substances hypotensives afin de déterminer leur mode d'action. Cette démarche recommande l'utilisation de techniques plus performantes tels que la microélectrode ou le patch clamp, et des préparations plus appropriées tels que les lambeaux d'aorte, et les muscles vasculaires.

L'utilisation de ces différentes techniques permettra une meilleure appréciation du mécanisme d'action des composantes cardiodépressives et hypotensives, à la fois dans les deux directions relatives à la vasodilatation liée à une action mécanique sur le diamètre des vaisseaux et à l'action cholinergique sur le centre cardiaque.

\section{Références}

[1] - E. S. ADJANOHOUN et ASSI AKE, "Contribution au recensement des plantes médicinales de Côte d'Ivoire", Centre National de Floristique de l'Université Nationale de Côte d'Ivoire. Tome I (1979), 356 pages.

[2] - A. BOUQUET et M. DEBRAY, "Plantes médicinales de Côte d'Ivoire", Travaux et documents de l'O.R.S.T.O.M N ${ }^{\circ}$ 32. O.R.S.T.O.M, Paris (1974), 231 pages.

[3] - A. ALVAREZ, F. POMAR, SEVILLA, M.J. MONTERO, J. Ethnopharmacol., 63 (3) (1999) 333-340.

[4] - T. M. Sarg, A. M. Ateya, N. M. Farrag, F.A. ABBas, Acta. Pharm. Hung., 61(6) (1991) 317-323.

[5] - P. GEISSBERGER, U. SEQUIN, Acta. Trop., 48 (4) (1991) 251-261.

[6] - R. L. PEREIRA, T. IBRAHIM, L. LUCCHETTI, A. J. DA SILVA, V. L. GONCALVES DE MORAS, Immunopharmacology, 43 (1) (1999) 31-37.

[7] - T. RABE, J. VAN STADEN, J. Ethnopharmacol., 56(1) (1997) 81-87.

[8] M. G. BRANDAO, A. U. KRETTLI, L. S. SOARES, G. NERY, C. MARINUZZIH, J. Ethnopharmacol., 57(2) (1997) 131-138.

[9] P. V. TAN, T. DIMO, E. DONG0, J. Ethnopharmacol., 73(3) (2000) 415-421.

[10] R. P. UBILLAS, C. D. MENDEZ, S. D. JOLAD, J. LUO, S. R. KING, T.J. CARLSON, D. M. FORT, Planta Med., 66 (1) (2000) 82-83.

[11] T. DIMO, RAKOTORINAS., R. KAMGANG, P. V. TAN, A. KAMANYI, M. BOPELET, J. Ethnopharmacol., 60 (2) (1998) 179-182.

[12] T. DIMO, T. B. NGUELEFACK, P. KAMTCHOUING, E. DONGO, A. RAKOTONIRINA, S. V. RAKOTONIRINA, CR. Acad. Sci. III, 322 (4) (1999) 323-329.

[13] H. W. CHIH, C. C. LIN, K.S. TANG, Am. J. Chin. Med., 24 (3-4) (1996) 231-40. 
[14] E. E. EHILE, A. MeNSAH-NYANGA, F. GUEde-gUINA et K. J. AKA, J. Ethnopharmacol., 33 (1991) 179-186.

[15] A. KamanYI, A. B. DONGm0, M. BOPElet, Rev. Méd. Pharm. Afr., 9 (2) (1995) 107-118.

[16] K. J-C. ABO, K. J. AKA, E. E. EHILE, F. GUEDE GUINA et F. TRAORE, Afrique Biomédicale, 5 (3) (2000) 11-20.

[17] R. G. BelemtOUGRI, C. N. mOUnAnga, Y. OUEdRa0G0, L. SAWAd0G0, Rev. Méd. Pharm. Afr., 15 (2001) 1-12.

[18] G. F. GUEDE, C. S. ISAI, M. 0. SMITH et R. F. OCHILLO, Afr. Biomed., 2 (2) (1997) 17-22.

[19] T. B. BOLTON, in "smooth muscle"., Ed. Ward Arnold, London (1981) 199-217.

[20] N. M. NATHANSON, A. Rev. Neurosci., 10 (1987) 195-236.

[21] A. PROKOPCZUK, B. LEWARTOWISKI and M. CZARNECKA, Pflügers Arch., 339 (1973) 305-316.

[22] W. R. GILES et S. J. NOBLE, J. physio/(Lond.), 261 (1976) 103-123.

[23] Y. IKEMOTO et M. GOTO, J. Molec. Cell. Cardiol., 9 (1977) 313-326.

[24] J. B. BASSINGTH WAIGHTE et H. REUTER, in "Electrical phenomena in the heart", Ed. De Mellow; C; New York, London : Academic press, (1972) 353-395.

[25] T. DIMO, A. RAKOTONIRINA, R. KAMGANG, T. R. TANKWA, K. W. G. J. M. WANDA, A. J. S. T., 2 (1) (2001) 32-35. 